\title{
Temperature-Sensitive poly(N-Isopropylacrylamide-Co-Butyl Methylacrylate) Nanogel as an Embolic Agent: Distribution, Durability of Vascular Occlusion, and Inflammatory Reactions in the Renal Artery of Rabbits
}

\author{
H. Zhao, C. Zheng, G. Feng, Y. Zhao, H. Liang, H. Wu, G. Zhou, B. Liang, Y. Wang, and X. Xia
}

\begin{abstract}
BACKGROUND AND PURPOSE: We have developed a new thermosensitive liquid embolic agent, PIB nanogel, that can be solidified at body temperature. We thus further investigated the distribution, durability of vascular occlusion, and inflammatory reactions of PIB in embolization of the renal artery of rabbits.

MATERIALS AND METHODS: The bilateral renal arteries of 9 rabbits were first embolized with PIB at different injection rates. The distribution pattern of PIB was investigated by contact radiography and histology 1 hour after embolization. The right renal arteries of 20 rabbits were then embolized with PIB at the proper injection rate. Angiography and pathologic examination of the kidneys were performed at 1 week and 1, 2, and 3 months after embolization to evaluate the long-term outcomes.

RESULTS: With the injection rate increasing, PIB could reach the more distal branch of the renal artery. The proper injection rate was chosen as $0.10 \mathrm{~mL} / \mathrm{s}$ due to the homogeneous distribution of PIB from the main renal artery to the precapillary level at this rate. During a 3-month follow-up observation period, no angiographic recanalization was observed. Histologically, we found no disruption of the vessel wall or subintimal bleeding, no extravasation of PIB, and no evidence of neovascularization. Moreover, there was only a mild inflammatory response, manifested by few lymphocytic and monocellular infiltration, without foreign body granuloma formation.
\end{abstract}

CONCLUSIONS: Embolization of the renal artery with PIB was easy and controllable, which could lead to a homogeneous and persistent occlusion without severe inflammatory changes. PIB might be a suitable material for intravascular embolization.

ABBREVIATIONS: EEL = external elastic lamina; EVG = Elastica-van Gieson; IEL = internal elastic lamina; LCST = lower critical solution temperature; PIB = poly( $N$-isopropylacrylamide-co-butyl methylacrylate); PNIPAM = poly( $N$-isopropylacrylamide)

$\mathbf{T}$ ranscatheter embolization plays an increasingly prominent role in interventional radiology and is widely used to treat various diseases, including hypervascular tumors, AVMs, and hemorrhages. ${ }^{1-3}$ A variety of embolic materials, including coils, particles, and liquid embolic agents, are used in intravascular embolotherapy based on the angioarchitectural and hemodynamic characteristic of the lesions. ${ }^{1-4}$ Currently, liquid embolic agents

Received November 24, 2011; accepted after revision March 30, 2012.

From the Department of Radiology (H.Z., C.Z., G.F., H.L., G.Z., B.L., Y.W., X.X.), Union Hospital, Tongji Medical College, and National Engineering Research Center for Nanomedicine (Y.Z.), College of Life Science and Technology, Huazhong University of Science and Technology, Wuhan, China; and Department of Radiology (H.W.), University Hospitals Case Medical Center, Case Western Reserve University, Cleveland, Ohio.

This study was supported in part by grants from 863 National High Technology Research and Development Program of China (No. 2006AA03Z332) and in part by National Natural Sciences Foundation of China (No. 30970804).

Please address correspondence to Chuansheng Zheng, Department of Radiology, Union Hospital, Tongji Medical College, Huazhong University of Science and Technology, 1277 Jiefang Rd, Wuhan, 430022, China; e-mail: hqzcsxh@sina.com

- Indicates open access to non-subscribers at www.ajnr.org

http://dx.doi.org/10.3174/ajnr.A3177 such as Onyx (ev3, Irvine, California) and cyanoacrylates have been widely and effectively used for many years. However, several problems have arisen, such as adhesiveness to the catheter and artery, complicated handling, and toxicity to living tissue. ${ }^{1,4,5}$ Therefore, it is necessary to search for an ideal embolic material to overcome these disadvantages.

Temperature-sensitive hydrogels can remain in liquid solution state at low temperature and become a gelatinous solid at temperatures above their LCST. Due to their nontoxicity, biocompatibility, and convenient administration, these have been extensively investigated for potential application in biomedical and pharmaceutical fields, including gene carriers, controlled drug delivery, and tissue engineering. ${ }^{6-9}$ However, only limited studies have focused on these polymers as embolic materials in the application of embolotherapy. Thermosensitive PNIPAM was first reported as an embolic agent by Matsumaru et al for intravascular neurosurgery. ${ }^{10}$ Poloxamer, which has rapid reversible sol-gel transition behavior with a change of temperature, was reported as a temporary embolic material several years later. ${ }^{11}$ Recently, a linear PNIPAM has been investigated in embolization of AVMs. ${ }^{12}$ 

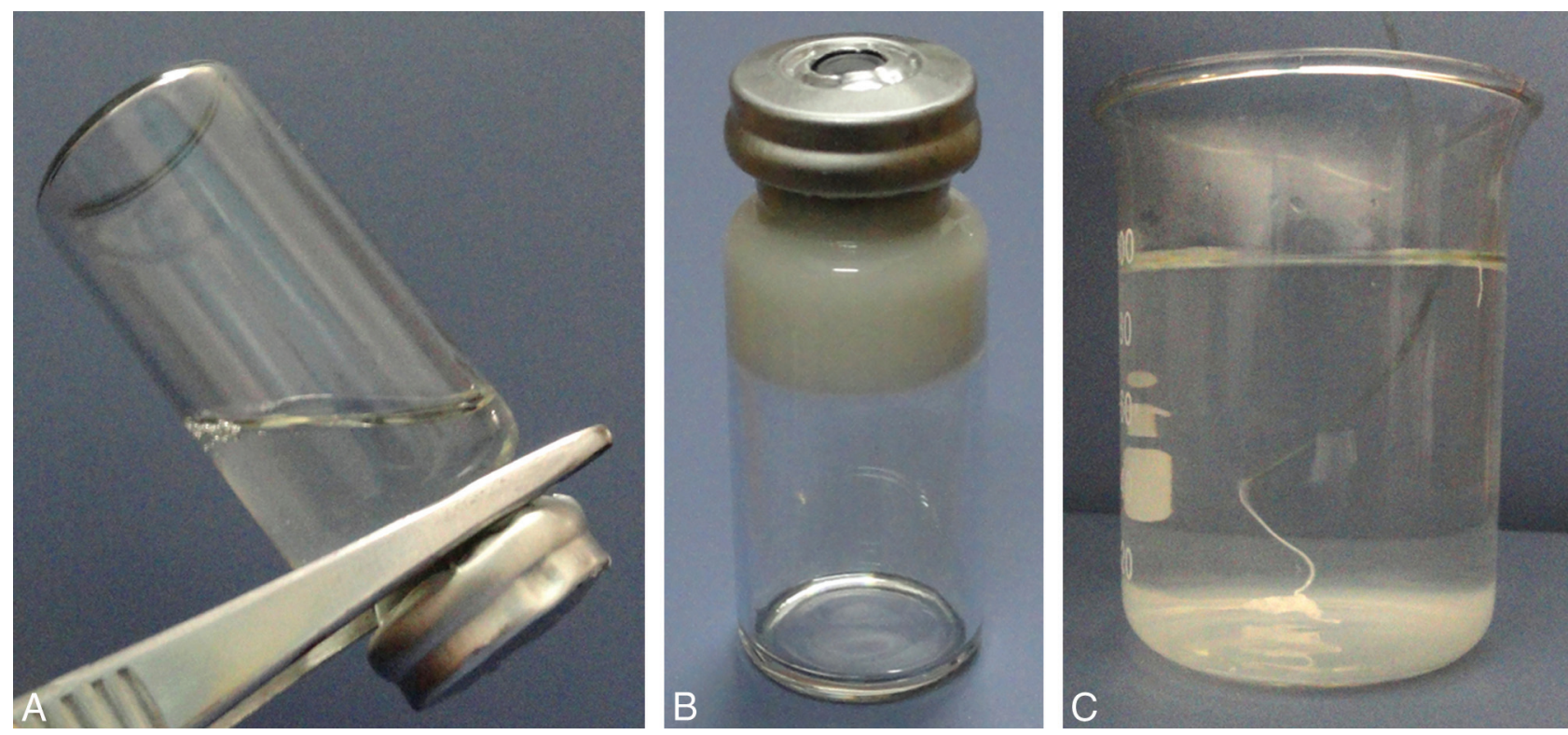

FIG 1. Characteristics of PIB. A, PIB is a flowable solution at low temperature, and (B) becomes a solid white gel at high temperature ( $\left.\geq 36^{\circ} \mathrm{C}\right) . \mathrm{C}$, $\mathrm{PIB}$ is injected into $37^{\circ} \mathrm{C}$ water through a $2.7 \mathrm{~F}$ microcatheter and rapidly solidifies as a white gel.

Nanogel (crosslinked polymer networks of $10-1000 \mathrm{~nm}$ ) dispersions have lower viscosity, higher strengths, faster gelation response rates, and stronger shear-thinning capacity compared with corresponding linear polymer solutions. ${ }^{13}$ Previously, we developed a PNIPAM nanogel, PIB, as a temperature-sensitive liquid embolic agent. ${ }^{13}$ Due to the presence of hydrophobic interactions above the volume phase transition temperature, these nanometer-sized gel particles can interconnect to form large networks. Compared with the hydrogels formed by corresponding linear polymers, these hierarchical 3D networks, composed of PNIPAM nanogel particles, have higher compressive strength. ${ }^{13}$ We propose that PIB nanogel is a potentially more durable thermosensitive liquid embolic agent.

The purpose of this animal study is to explore the optimal injection technique, feasibility, and effectiveness of PIB nanogel in intravascular embolization. Using a rabbit kidney endovascular embolization model, a well-known and intensively investigated model for studying vascular embolization, ${ }^{14-17}$ we first sought to determine an ideal embolization methodology by investigating the depth of penetration and distribution characteristics of PIB with various injection rates. We then observed the long-term tissue inflammatory reactions and the durability of embolization over 3 months.

\section{MATERIALS AND METHODS \\ Embolic Material}

PIB nanogel was prepared via precipitation polymerization, as previously reported. ${ }^{13}$ Briefly, $N$-isopropylacrylamide (2.26 g), butyl methylacrylate $(0.14 \mathrm{~g}), N, N^{\prime}$-methylenebisacrylamide $(0.03 \mathrm{~g})$, and sodium dodecyl sulfate $(0.03 \mathrm{~g})$ were dissolved in water $(180 \mathrm{~mL})$. The resulting solutions were degassed for at least 60 minutes by bubbling $\mathrm{N}_{2}$ and heating to $70^{\circ} \mathrm{C}$ with stirring, followed by addition of potassium persulfate $(95 \mathrm{mg})$ as an initiator for the polymerization. The reaction continued under an $\mathrm{N}_{2}$ at $70^{\circ} \mathrm{C}$ for 4.5 hours. The obtained PIB nanogel dispersions were purified by dialysis for 2 weeks to remove unreacted monomers and impurities. PIB nanogel dispersions were lyophilized, sterilized, and stored at room temperature.

To achieve appropriate radiopacity, iohexol (300 mg of I/mL) was prediluted to $50 \%$ with distilled water and then $6 \%$ (wt $/ \mathrm{wt}$ ) PIB solution was prepared in the diluted iohexol solution under strict sterile conditions, as previously reported. ${ }^{13}$ The size of the PIB nanogel particle is $100-400 \mathrm{~nm}$, the LCST is $36.5^{\circ} \mathrm{C}$, and other physicochemical properties have been well studied in our previous report. ${ }^{13}$ The PIB dispersion underwent a sol-gel transition at its LCST of $36.5^{\circ} \mathrm{C}$ (Fig $1 A,-B$ ). As Fig $1 C$ shows, the dispersion turned into a white gel within 3 seconds when injected into $37^{\circ} \mathrm{C}$ water through a $2.7 \mathrm{~F}$ microcatheter.

\section{Embolization Procedures}

The study was approved by the Animal Care Committee of our institution and experiments were performed in accordance with institutional guidelines. A total of 29 adult Japanese white rabbits weighing $2.5-3.0 \mathrm{~kg}$ were used. The animals were fed under standard conditions in the research laboratory and starved for 12 hours before each procedure. For anesthesia, sodium pentobarbital $(30 \mathrm{mg} / \mathrm{kg})$ was used intravenously.

Interventional procedures were performed using a DSA unit and strict sterile technique. The right femoral artery was surgically isolated and accessed through open puncture with an 18-gauge sheath needle. A 4F arterial sheath was then placed into the femoral artery, and sodium heparin (100 IU/kg) was administered using the side arm. A 4F Cobra visceral catheter (Terumo, Tokyo, Japan) was inserted in the orifice of the renal artery, and a preembolization renal arteriogram was obtained by injecting $2 \mathrm{~mL}$ of contrast media.

A 2.7F microcatheter was inserted into the $4 \mathrm{~F}$ catheter through a hemostatic Y valve, which had been connected to the hub of the $4 \mathrm{~F}$ catheter, and gently advanced to the main renal artery as distal 
as possible to perform embolization. To prevent PIB from solidifying, resulting from body temperature in the microcatheter during delivery, the $Y$ valve was continuously flushed with cold saline $\left(10^{\circ} \mathrm{C}\right)$ via an infusion pump. Under fluoroscopic monitoring, PIB was injected using a syringe pump to obtain better control. When the material began to reflux toward the microcatheter (embolization end point), the injection was halted and infusion of cool saline was suspended. The microcatheter was removed and the remaining polymer in the microcatheter was washed out with cold saline. After careful injection of $1 \mathrm{~mL}$ of contrast media to determine whether vasospasm was present, the $4 \mathrm{~F}$ catheter was removed. To avoid angiographic misinterpretation from early redistribution of PIB, we did not perform a postembolization renal arteriogram via the same Cobra catheter until after a waiting period of 10 minutes. ${ }^{14,18}$ After the procedure, the catheter and sheath were removed and the femoral artery was tied.

\section{Experiment 1: Selection of a Proper Injection Rate}

Nine animals were used in this study, evenly divided into 3 groups. In each group, the PIB was injected into bilateral renal arteries at a rate of $0.05 \mathrm{~mL} / \mathrm{s}, 0.10 \mathrm{~mL} / \mathrm{s}$, or $0.15 \mathrm{~mL} / \mathrm{s}$, respectively. One hour after the embolization procedure, the animal was euthanized with an overdose of sodium pentobarbital (100 mg/ $\mathrm{kg}$ ). Macroscopic inspection of the abdominal space and thoracic cavity was performed to search for possible complications of embolization. The kidneys were harvested and contact radiography was performed to evaluate the presence and distribution of PIB.

For histopathologic preparation, each kidney was cut longitudinally through the hilum into 2 halves. Then, 3 radial $3-\mathrm{mm}$ thick sections from the upper, medium, and lower poles were sectioned from each kidney half, traversing the hilum to the cortex. Each section was fixed with $10 \%$ neutral buffered formalin at $37^{\circ} \mathrm{C}$ (the polymer liquefies easily at lower temperatures), embedded in paraffin, sectioned at $4 \mu \mathrm{m}$, and stained with EVG, a specific staining method used for the study of vascular architecture, and hematoxylin-eosin.

The histologic analyses mainly focused on the distribution characteristics of PIB, including the homogeneity and completeness of vascular penetration. Close attention was paid to determine the occlusion level by investigating the embolized renal artery level. In this regard, the arterial vessels were categorized according to their locations, sizes, and wall structures in histologic sections.

\section{Experiment 2: Durability of Embolization, Degree of Vascular Wall Injury, and Inflammatory Reactions}

After having determined the proper injection rate of PIB, we further investigated the embolization efficacy by evaluating the durability of embolization and biocompatibility of PIB in the right kidney of 20 rabbits with the proper injection rate of PIB. Rabbits were divided into 4 groups by the timing of sacrificing after embolization: 1 week, and 1, 2, and 3 months, with 5 rabbits in each group. Before sacrificing, the animal first underwent a follow-up renal angiogram, which was performed via left groin for vascular access (as described in the pre-embolization renal arteriogram procedure), to evaluate recanalization. The macroscopic inspec- tion and section and staining of kidney samples were the same as those described in experiment 1.

The parameters and grading criteria of histologic analysis of the kidney specimen consisted of the following:

1) Intraluminal and perivascular inflammation: none, mild, moderate, or severe by the degree of cellular responses and the specific cell types present.

2) PIB-induced vascular injury: classified using the Schwartz $\operatorname{method}^{15,19}$ : none, IEL intact; mild, disruption of IEL without medial disruption; moderate, disruption of IEL and media without EEL disruption; and severe, disruption of IEL, media, and EEL.

3) Mural hemorrhage, extravasation, and neovascularization: rated as absent or present. Extravasation was noted as the presence of embolic agent outside the vessel lumen. ${ }^{14,20}$ Neovascularization was defined as the newly developed endothelialized channels in the previously embolized lumen. ${ }^{14,15}$

\section{RESULTS}

\section{Embolization Procedure and Animal Survival}

PIB nanogel could be visualized under fluoroscopy, due to the content of iohexol, and the behavior of PIB could be monitored during embolization. With the described technique, the injection of PIB was easily controlled. A gradual decrease of dispersion velocity of PIB was noted, which might be due to the gradual gelatification of PIB under exposure to body temperature. There was no adhesion of the microcatheter to the vessel or occlusion of the microcatheter. Furthermore, no vascular spasm was observed during the whole embolization procedure. No procedure-related complications were noted and all animals survived without complications until sacrifice. The volume amount (expressed as mean \pm standard deviation) of PIB for embolization in each group was $0.47 \pm 0.08 \mathrm{~mL}(n=6 ; 0.05-\mathrm{mL} / \mathrm{s}$ group $), 0.85 \pm 0.09$ $\mathrm{mL}(n=6 ; 0.10-\mathrm{mL} / \mathrm{s}$ group $)$, and $0.98 \pm 0.12 \mathrm{~mL}(n=6 ; 0.15-$ $\mathrm{mL} / \mathrm{s}$ group $)$ in experiment 1 ; and $0.86 \pm 0.12 \mathrm{~mL}(n=5$; 1 -week group), $0.84 \pm 0.09 \mathrm{~mL}(n=5 ; 1$-month group), $0.87 \pm 0.11 \mathrm{~mL}$ $(n=5$; 2-month group), and $0.83 \pm 0.10 \mathrm{~mL}(n=5$; 3 -month group) in experiment 2 .

\section{Radiographic and Pathologic Findings of Experiment 1}

$\mathrm{PIB}$, visualized by contact radiograph as a cast in the renal arteries, distributed homogeneously in embolized kidneys (Fig 2). As shown in Fig $2 A$, a low injection rate $(0.05 \mathrm{~mL} / \mathrm{s})$ resulted in the embolization of segmental arteries and proximal interlobar arteries. With an increasing injection rate $(0.10 \mathrm{~mL} / \mathrm{s}), \mathrm{PIB}$ distribution became wider (Fig $2 B$ ), penetrating the peripheral vessels at the injection rate of $0.15 \mathrm{~mL} / \mathrm{s}$ (Fig 2C).

Apart from blanched kidneys, no abnormal change was observed by the naked eye in other organs before histologic studies. Figure 3 shows the microscopic views of the kidneys embolized by PIB at various injection rates. PIB appeared as amorphous material within vessels. PIB was located mainly in the segmental and interlobar arteries at a low injection rate (Fig $3 A$ ), while it arrived at arcuate and interlobular arteries $(50-150 \mu \mathrm{m}$ in diameter) at a higher injection rate (Fig $3 B$ ) and further reached glomerular capillaries as small as $5 \mu \mathrm{m}$ in diameter at the highest injection rate (Fig 3C). The levels of the embolized renal artery at different in- 

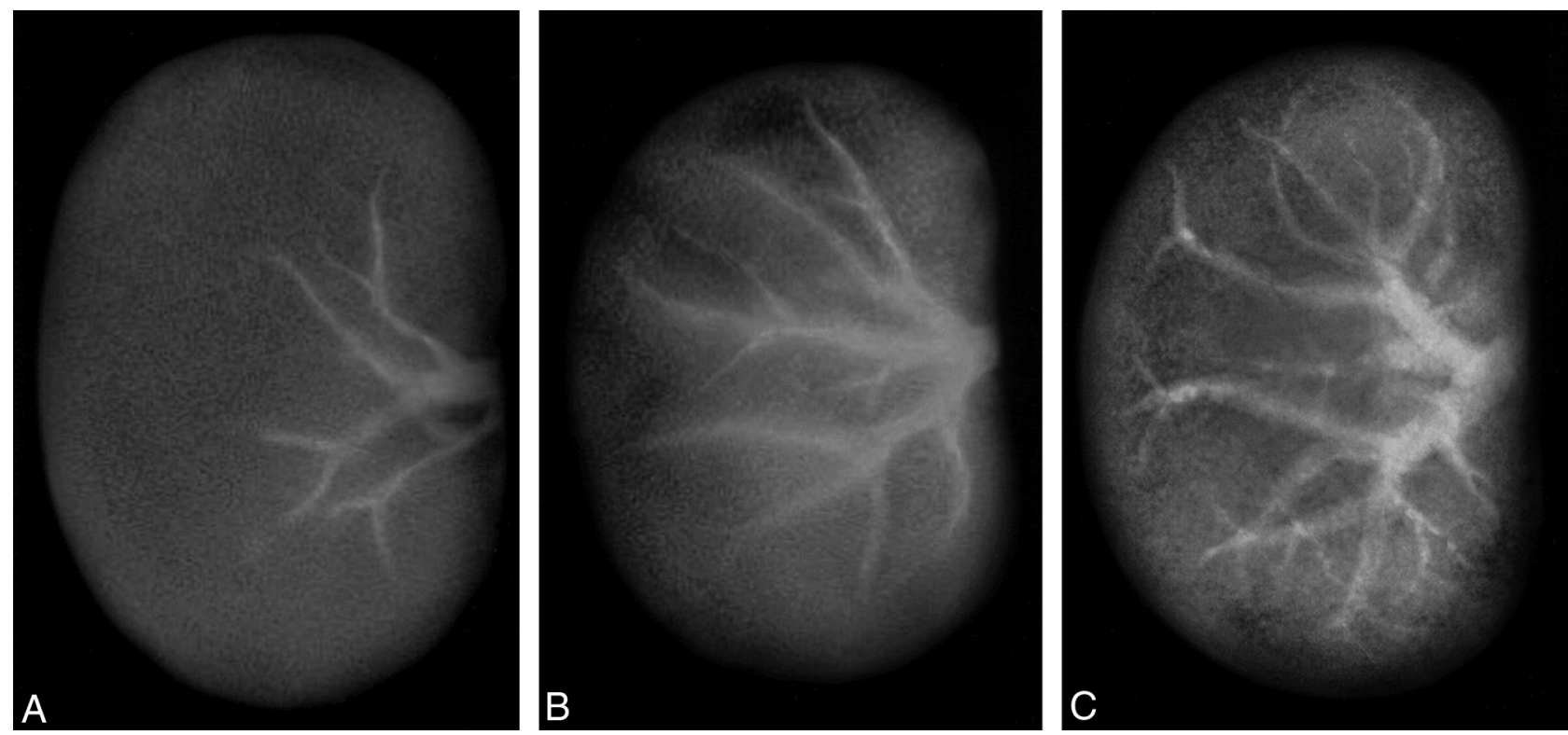

FIG 2. Contact radiographs of the kidneys embolized by PIB at various injection rates. $A$, PIB with low injection rate $(0.05 \mathrm{~mL} / \mathrm{s})$ reaches the proximate segmental and interlobar arteries. $B$, With increasing of injection rate $(0.10 \mathrm{~mL} / \mathrm{s})$, distribution becomes wider. $C$, PIB with high injection rate $(0.15 \mathrm{~mL} / \mathrm{s})$ penetrates the peripheral vessels.
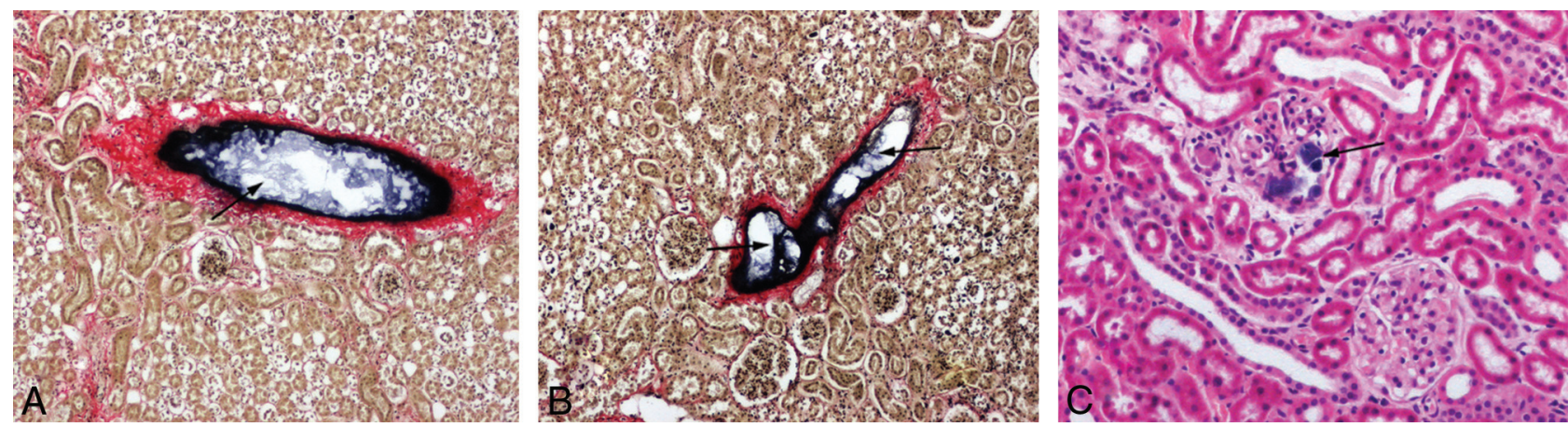

FIG 3. Microscopic views of the kidneys embolized by PIB (arrows in the figures) at various injection rates. $A$, PIB with low injection rate ( 0.05 $\mathrm{mL} / \mathrm{s}$ ) only reaches the interlobar artery, and $(B)$ that with $0.10 \mathrm{~mL} / \mathrm{s}$ locates in the interlobular and arciform arteries (the empty spaces in the embolized lumen are due to dissolution of the copolymer during processing and staining of the histologic specimen at room temperature) (EVG stain, $\times 100$ ). C, PIB with $0.15 \mathrm{~mL} / \mathrm{s}$ within glomerular capillaries (hematoxylin-eosin stain, $\times 200$ ). Neither vascular injury nor hemorrhagic change is observed.

Table 1: Occlusion level of kidney vessels at different injection rates

\begin{tabular}{ll}
$\begin{array}{c}\text { Injection } \\
\text { Rate }(\mathrm{mL} / \mathrm{s})\end{array}$ & \multicolumn{1}{c}{ Embolized Renal Artery Level } \\
\hline 0.05 & Segmental and interlobar arteries \\
0.10 & $\begin{array}{c}\text { Arcuate and interlobular arteries } \\
\text { Interlobular arteries, afferent arterioles, and glomerular } \\
\text { capillaries }\end{array}$ \\
\hline
\end{tabular}

jection rates are summarized in Table 1 . These findings regarding the uniform distribution of PIB and its relationship with injection rate were consistent with the results from the distribution area of PIB from radiologic studies (Fig 2).

Although no venous embolization was observed in the 0.15$\mathrm{mL} / \mathrm{s}$ group, the occlusion of glomerular capillaries suggested increased incidence of nanogel particles passing through the glomeruli and entering the venous side. Thus, the rate of $0.10 \mathrm{~mL} / \mathrm{s}$ was selected to optimize the embolizing effect and avoid venous embolization.

\section{Angiographic and Pathologic Findings of Experiment 2}

The immediate postembolization arteriograms of all groups showed complete occlusion of the renal artery. Angiograms obtained from 1-week, and 1-, 2-, and 3-month follow-up demonstrated persistence of occlusion without the evidence of recanalization or nontarget embolization. Immediate and 3-month postembolization angiograms of a typical animal are shown in Fig 4.

After embolization, a tendency of shrinkage of the embolized kidney, together with compensatory hypertrophy of the contralateral kidney, without evidence of ischemia and infarction in the lung, bowel, and adrenal gland, was noted by macroscopic observations at each follow-up observation time point. In addition, there were no cases of abscess formation in any treated kidneys. Firm adhesion between the yellowish kidney and perirenal reactive tissue, and irregular as well as dispersed small blood vessels in the nodular surfaces of the embolized kidney, were observed at 1 week and 1 month. At 2 and 3 months, uniform shrink- 


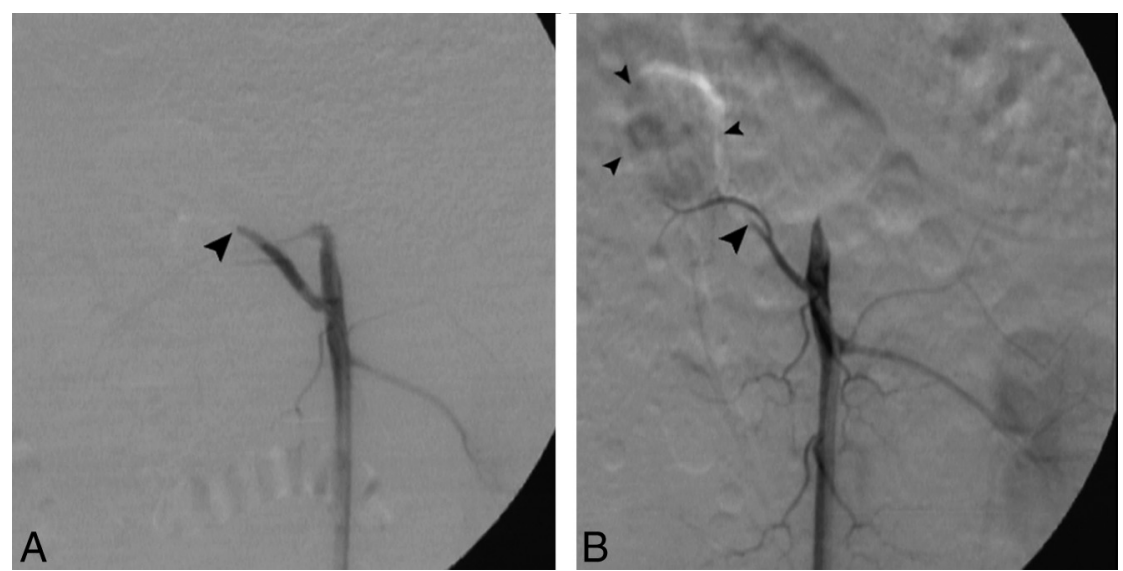

FIG 4. Selective right renal arterial angiograms. A, Angiogram obtained 10 minutes after embolization with PIB shows complete occlusion of the right main renal artery (large arrowhead). $B$, Three months after embolization, the right main renal artery (large arrowhead) remains totally occluded and the affected kidney (small arrowheads) shows marked shrinkage.
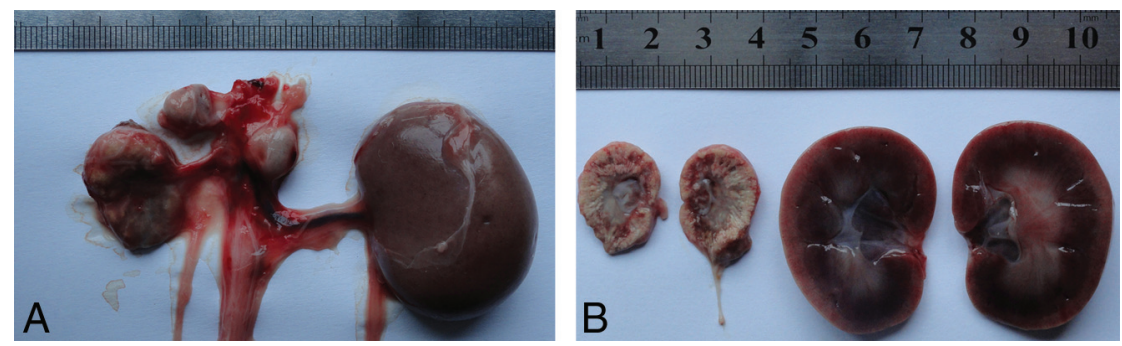

FIG 5. Gross outside and section views of the embolized and control kidney at 3 months after embolization with PIB showing notable atrophy $(A)$, and calcification $(B)$ of the affected kidney compared with the left untreated kidney.

age and calcification was noted in the affected kidney. Moreover, vague demarcation between cortex and medulla of the embolized kidney was also observed. Representative images taken from an embolized kidney in group 4 are shown in Fig 5 .

The histologic findings are summarized in Table 2. PIB was evident in all groups without occurrence in any venule or vein. Moreover, no extraluminal migration of PIB was observed, which was consistent with our findings that there was no vascular injury or mural hemorrhage, and supported our data that no neovascularization in the embolized vessels was found. Mild inflammation, demonstrated as presence of neutrophils and mononuclear cells (though fewer than neutrophils) in and around the embolized vessels, was observed at 1 week. However, at 1 month and 2 months, infiltration of inflammatory cells, mainly composed of lymphocytes, neutrophils, and mononuclear cells, was less evident than that at 1 week. Moreover, the embolized vessels were filled with PIB and fibrous tissues without neovascularization. It was important to observe that PIB was still found in the vessels without degradation and inducing inflammatory reactions at 3 month. Notably, no foreign body granuloma was observed in the specimens of any groups (Fig 6).

\section{DISCUSSION}

Hydrogels have been used as embolic materials because they are nonadhesive to surrounding tissue and can be loaded with drugs..$^{21}$ Matsumaru et al ${ }^{10}$ first reported thermosensitive PNIPAM hydrogels as an embolic agent to embolize rabbit kidneys in 1996. However, the embolic material used in their study is nonradiopaque. In embolotherapy, the hydrogels must be combined with an $\mathrm{x}$-ray contrast agent (eg, iohexol) for monitoring and evaluating the embolization. Previously, we synthesized thermosensitive nanogels composed of PNIPAM with and without butyl methylacrylate, and investigated the effect of incorporation of iohexol on their sol-gel phase transition behavior. ${ }^{13}$ Our previous data indicated that the dispersion process of pure PNIPAM nanogel in iohexol raised its LCST to $46^{\circ} \mathrm{C}$, which resulted in no gelation at body temperature. ${ }^{13}$ This makes it unsuitable for an in vivo test. In contrast, PIB nanogel can homogeneously embolize renal arteries of different sizes at different injection rates as shown in this study. PIB, with its nonadhesiveness, good biocompatibility, and radiopacity (with iohexol), at an injection rate of $0.10 \mathrm{~mL} / \mathrm{s}$, can produce rapid, complete, and effective occlusion of the vessels in a kidney model with a 3-month follow-up.

\section{Distribution of PIB}

The arterial occlusion level and distribution pattern of an embolic material is of great clinical significance to avoid nontarget embolization and to prevent serious complications. ${ }^{1}$ In this study, we found that the level of embolized vessels was related to the injection rate. A high injection rate would cause rapid distribution of PIB into the peripheral vessels of the renal parenchyma, due to the high impact and propulsion force. A lower rate, however, might lead to more gelation response in the proximal portion of the renal artery. Nevertheless, an abnormally high injection rate could result in reflux of PIB and/or PIB going through to the venous side, both of which could lead to catastrophic nontarget embolization. As mentioned previ-

Table 2: Histologic findings in arteries embolized with PIB

\begin{tabular}{llllccc}
\hline Follow-Up & $\begin{array}{l}\text { Intraluminal } \\
\text { Inflammation }\end{array}$ & $\begin{array}{c}\text { Perivascular } \\
\text { Inflammation }\end{array}$ & $\begin{array}{c}\text { Vascular } \\
\text { Injury }\end{array}$ & $\begin{array}{c}\text { Mural } \\
\text { Hemorrhage }\end{array}$ & Extravasation & Neovascularization \\
\hline 1 week & Mild & Mild & None & Absent & Absent & Absent \\
1 month & None or mild & Mild & None & Absent & Absent & Absent \\
2 months & None or mild & None or mild & None & Absent & Absent & Absent \\
3 months & None & None & None & Absent & Absent & Absent \\
\hline
\end{tabular}


ously, a proper injection rate must be carefully selected. We chose $0.10 \mathrm{~mL} / \mathrm{s}$ as the standard rate, as PIB was shown to reach the precapillary level of kidney at this rate.

Under tested injection rates, we found that PIB was distributed relatively homogeneously within the entire renal arterial system. This could be explained by its homogeneous attenuation and the fact that no vascular spasm was induced at various injection rates in our experiment. The attenuation of PIB was $1.10 \mathrm{~g} / \mathrm{mL}$, similar to that of the whole blood. This would thus make PIB flow in a uniform pattern in vessels. ${ }^{22}$ Vascular spasm, mainly caused by the embolic material or the catheterization, can increase the risk of incomplete and inhomogeneous embolization. ${ }^{16,18}$ No vascular spasm was noted in this study, which highlights the notion that PIB might be an ideal material for embolization.

In addition to injection rate, occlusion level of PIB might also be controlled by the position of the microcatheter. During the operation, the inner microcatheter was continuously flushed with cool saline through the outer Cobra catheter. This method has advantages for both slow-flow and highflow vessels. For tortuous vessels or vessels with slow blood flow, the microcatheter could be retracted behind of the catheter. The cool saline allowed PIB to flow farther downstream before gelling and thus made PIB better fill the vessels. For high-flow vessels, such as AVMs, the microcatheter could be advanced ahead of the catheter so that the gelation began within the head of microcatheter. This procedure would lead to a stable gel mass that could quickly occlude higher-flow systems with minimal gel penetration or concern for downstream propagation. However, the influence of the position of the microcatheter on occlusion level of PIB warrants future research for verification.

As mentioned previously, the blood flow conditions at the injection site also influenced the occlusion level of PIB. The renal artery model used in this study is distinct from AVMs, which have blood flow of higher volume and velocity. The distribution of PIB in the renal artery might not be simply extended to the general embolization of AVMs, due to the different angioarchitectural and hemodynamic characteristics. Further studies are needed to determine whether injection of PIB is capable of producing a safe and effective AVM nidus occlusion.

\section{Recanalization}

Recanalization is a frequently reported phenomenon in embolization of vascular malformations and tumors. Collateralization,
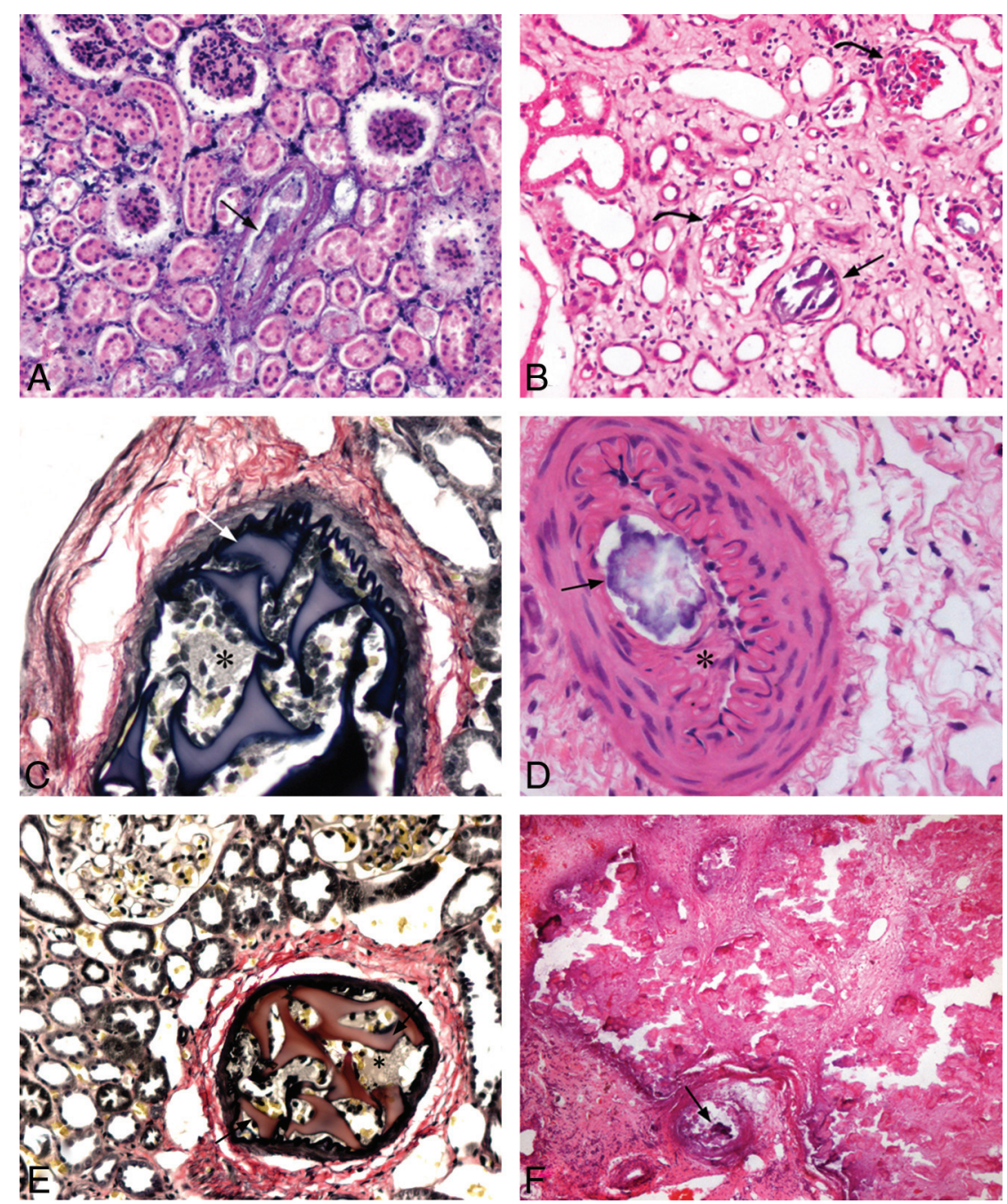

FIG 6. Microscopic views of the embolized kidneys at 1 week $(A), 1$ month $(B, C), 2$ months $(D, E)$ and 3 months $(F)$ after the injection of PIB (arrows in the figures). $A$, The occluding plug consists almost exclusively of PIB, with mild inflammatory reaction (hematoxylin-eosin stain, $\times 200$ ). B, Atrophic glomeruli (curved arrows) are surrounded by sclerotic tissues. Intraluminal PIB and vessel walls are

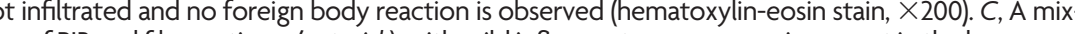
of PIB and fibrous tissue (asterisk), with mild inflammatory response, is present in the lumen and ( without signs of neovascularization or mural hemorrhage (hematoxylin-eosin stain, $\times 400$ ). E, The the arterial wall remains intact (EVG stain, $\times 400$ ). F, PIB remains inside the vessel without extravasation or cellular infiltration. The necrotic, fibrotic, and calcific kidney tissues are seen throughout in the view (hematoxylin-eosin stain, $\times 100$ ).

which occurs via shunt opening or recruitment of nonembolized vessels to supply an embolized and subsequent ischemic area, would increase the incidence of recanalization. This mechanism has been described in embolization of porcine rete mirabile and polar renal artery. ${ }^{21,23,24}$ Different from these studies, we embolized the main renal artery of a rabbit, which might decrease the incidence of recanalization, possibly due to the characteristic anatomy of terminal circulation. ${ }^{14}$ The possibility of recanalization after embolization of organs with collateral vascular supply warrants further research. Nevertheless, our results that 1) no recanalization was observed in angiograms obtained up to 3 months after embolization, and 2) no neovascularization of the embolized vessels was demonstrated histologically, indicate that PIB may be an ideal embolization material. 
There are reasons that might contribute to nonrecanalization after PIB embolization. One is that PIB is a nonbiodegradable material (due to the nonbiodegradable component of PNIPAM), which rules out the possibility of the degradation of the embolus. In addition, vascular spasm, which is a risk factor of incomplete embolization as well, ${ }^{16,18}$ was not observed. A frequently reported reason for recanalization was that the embolus might be redistributed by arterial pulsation or increased pressure in the feeder due to embolization. ${ }^{21}$ Because of the hierarchical nanogel networks formed by the temperature-sensitive sol-gel phase transition, the gel embolus of PIB was stable and could effectively resist blood underscouring. Furthermore, the compressive strength of this hierarchical 3D network could be further enhanced by the hydrogen bonding between iohexol and nanogel particles. ${ }^{13}$ Another frequent reason for recanalization is extravasation of the embolic material due to vessel wall destruction. This mechanism has been described in previous reports, including embolization of human AVMs with cyanoacrylates ${ }^{25}$ or polyvinyl alcohol particles, ${ }^{26}$ and embolization of the animal kidney ${ }^{14,27}$ or uterus ${ }^{20}$ with polyvinyl alcohol particles and various microspheres. Because no vessel wall damage was observed in our study, this also contributes to the observed results.

\section{Inflammation Reactions}

We observed a gradually declining inflammatory severity from 1 week to 3 months after operation. Because no signs of bacterial colonization were noted, the intraluminal and perivascular inflammation might be due to the toxic effects of ischemia and the embolic material itself. ${ }^{15,27}$ In our experiment, the inflammatory changes may partly result from the ischemic effect of the embolization of the kidney that has a terminal circulation without collateral vascular supply. Embolization could produce an ischemic necrosis of kidney tissue that induced various degrees of inflammation reactions trying to "clean" the damaged tissue. ${ }^{24}$

As mentioned above, inflammation could also be induced by the embolic material. For liquid embolics, the biocompatibility is related to the phase change from liquid to solid and the nature of the material. ${ }^{4}$ Precipitating liquid embolics (eg, ethylene-vinyl alcohol copolymers) was always dissolved in organic solvent (eg, dimethyl-sulfoxide) to perform embolization, but the organic solvent had been reported to induce high toxicity with vascular failure, bleeding, and vascular necrosis in animal experiments. ${ }^{5,28,29}$ Another reason for material-related toxicity is attributed to the heat generated during polymerization and release of toxic components (acrylacetate and formaldehyde) for polymerizing liquid embolics (eg, cyanoacrylates). ${ }^{1,4,5}$ Unlike these materials, PIB does not need organic solvent in preparation, making it perfect for avoiding the related potential toxicity. It is a thermosensitive copolymer and becomes a gelatinous solid at body temperature without releasing heat and toxic components. ${ }^{13}$ Hence, the characteristics of PIB are advantageous in that they are devoid of the material-related toxicities mentioned above.

Importantly, PIB is a nondegradable material that could cause a milder inflammatory reaction than rapidly and slowly degradable materials. ${ }^{2}$ Notably, a foreign body reaction (giant cells) was not observed in the embolized vessels or surrounding tissues at 3 months. This further supports the good biocompatibility of PIB.
Additionally, a mixture of PIB and fibrous tissue was found in the occluded vessels. It has been reported that nondegradable materials may cause a milder inflammatory reaction that could progress to fibrosis, ${ }^{2}$ and this fibrotic reaction might help with the persistence of the embolic effect. ${ }^{17}$

Water solubility, nonadhesiveness, good biocompatibility, efficient embolization, and thermoreversibility are all advantages of PIB. As thermoreversibility allows PIB to be redissolved when the temperature falls, nontargeted embolization of PIB could be solved via redissolution, ${ }^{30}$ and PIB might also be used for temporary embolization. ${ }^{11}$ Because PNIPAM-based polymers have been shown to serve as vectors for drugs, proteins, and target genes, ${ }^{6-8}$ PIB could also be modified to a "bioactive embolic agent" and thus widely applied in endovascular therapy.

\section{CONCLUSIONS}

PIB nanogel is nonadhesive, contains no organic solvents, and has a well-defined starting and ending point of embolization because it is radiopaque (with iohexol). Embolization of renal arteries with PIB is easy and controllable. The occlusion level of kidney vessels can be adjusted by adjusting the injection rate. PIB can result in homogeneous and durable occlusion of the vessels without severe inflammatory reactions at an injection rate of 0.10 $\mathrm{mL} / \mathrm{s}$ in a 3-month follow-up. PIB could be developed as a thermosensitive embolic material for treating tumors and AVMs, and offers potential for future developments in embolic design.

\section{ACKNOWLEDGMENTS}

We thank Professor Xiangliang Wang, Chief of National Engineering Research Center for Nanomedicine, Huazhong University of Science and Technology, for advice on preparing the PIB dispersions.

\section{REFERENCES}

1. Pollak JS, White RI Jr. The use of cyanoacrylate adhesives in peripheral embolization. J Vasc Interv Radiol 2001;12:907-13

2. Loffroy R, Guiu B, Cercueil JP, et al. Endovascular therapeutic embolisation: an overview of occluding agents and their effects on embolised tissues. Curr Vasc Pharmacol 2009;7:250-63

3. Lewandowski RJ, Geschwind JF, Liapi E, et al. Transcatheter intraarterial therapies: rationale and overview. Radiology 2011;259: 641-57

4. Jordan O, Doelker E, Rufenacht DA. Biomaterials used in injectable implants (liquid embolics) for percutaneous filling of vascular spaces. Cardiovasc Intervent Radiol 2005;28:561-69

5. Bakar B, Oruckaptan HH, Hazer BD, et al. Evaluation of the toxicity of Onyx compared with n-butyl 2-cyanoacrylate in the subarachnoid space of a rabbit model: an experimental research. Neuroradiology 2010;52:125-34

6. Zhou YM, Ishikawa A, Okahashi R, et al. Deposition transfection technology using a DNA complex with a thermoresponsive cationic star polymer. J Control Release 2007;123:239-46

7. Sabnis A, Wadajkar AS, Aswath P, et al. Factorial analyses of photopolymerizable thermoresponsive composite hydrogels for protein delivery. Nanomedicine 2009;5:305-15

8. Wang Q, Zhao YB, Yang YJ, et al. Thermosensitive phase behavior and drug release of in situ gelable poly ( $\mathrm{N}$-isopropylacrylamide-coacrylamide) microgels. Colloid Polym Sci 2007;285:515-21

9. Klouda L, Mikos AG. Thermoresponsive hydrogels in biomedical applications. Eur J Pharm Biopharm 2008;68:34-45

10. Matsumaru Y, Hyodo A, Nose T, et al. Application of thermosensi- 
tive polymers as a new embolic material for intravascular neurosurgery. J Biomater Sci Polym Ed 1996;7:795-804

11. Raymond J, Metcalfe A, Salazkin I, et al. Temporary vascular occlusion with poloxamer 407. Biomaterials 2004;25:3983-89

12. Li X, Liu W, Ye G, et al. Thermosensitive $\mathbf{N}$-isopropylacrylamide$\mathrm{N}$-propylacrylamide-vinyl pyrrolidone terpolymers: synthesis, characterization and preliminary application as embolic agents. Biomaterials 2005;26:7002-11

13. Zhao $\mathrm{Y}$, Zheng C, Wang Q, et al. Permanent and peripheral embolization: temperature-sensitive $\mathbf{p}(N$-isopropylacrylamide-cobutyl methylacrylate) nanogel as a novel blood-vessel-embolic material in the interventional therapy of liver tumors. Adv Funct Mater 2011;21:2035-42

14. Senturk C, Cakir V, Yorukoglu K, et al. Looking for the ideal particle: an experimental embolization study. Cardiovasc Intervent Radiol 2010;33:336-45

15. Kwak BK, Shim HJ, Han SM, et al. Chitin-based embolic materials in the renal artery of rabbits: pathologic evaluation of an absorbable particulate agent. Radiology 2005;236:151-58

16. Kónya A, Van Pelt CS, Wright KC. Ethiodized oil-ethanol capillary embolization in rabbit kidneys: temporal histopathologic findings. Radiology 2004;232:147-53

17. Sadato A, Wakhloo AK, Hopkins LN. Effects of a mixture of a low concentration of n-butylcyanoacrylate and ethiodol on tissue reactions and the permanence of arterial occlusion after embolization. Neurosurgery 2000;47:1197-203; discussion 1204-05

18. Klisch J, Yin L, Requejo F, et al. Liquid 2-poly-hydroxyethyl-methacrylate embolization of experimental arteriovenous malformations: feasibility study. AJNR Am J Neuroradiol 2002;23: 422-29

19. Schwartz RS, Huber KC, Murphy JG, et al. Restenosis and the proportional neointimal response to coronary artery injury: results in a porcine model. J Am Coll Cardiol 1992;19:267-74

20. Laurent A, Wassef M, Namur J, et al. Recanalization and particle exclusion after embolization of uterine arteries in sheep: a longterm study. Fertil Steril 2009;91:884-92
21. Gobin YP, Vinuela F, Vinters HV, et al. Embolization with radiopaque microbeads of polyacrylonitrile hydrogel: evaluation in swine. Radiology 2000;214:113-19

22. Kai Y, Hamada J, Morioka M, et al. The utility of the microcrystalline cellulose sphere as a particulate embolic agent: an experimental study. AJNR Am J Neuroradiol 2000;21:1160-63

23. Arakawa H, Murayama Y, Davis CR, et al. Endovascular embolization of the swine rete mirabile with Eudragit-E 100 polymer. AJNR Am J Neuroradiol 2007;28:1191-96

24. de Luis E, Bilbao JI, de Ciercoles JA, et al. In vivo evaluation of a new embolic spherical particle (HepaSphere) in a kidney animal model. Cardiovasc Intervent Radiol 2008;31:367-76

25. Vinters HV, Lundie MJ, Kaufmann JC. Long-term pathological follow-up of cerebral arteriovenous malformations treated by embolization with bucrylate. $N$ Engl J Med 1986;314:477-83

26. Germano IM, Davis RL, Wilson CB, et al. Histopathological follow-up study of 66 cerebral arteriovenous malformations after therapeutic embolization with polyvinyl alcohol. J Neurosurg 1992; 76:607-14

27. Bilbao JI, de Luis E, Garcia de Jalon JA, et al. Comparative study of four different spherical embolic particles in an animal model: a morphologic and histologic evaluation. J Vasc Interv Radiol 2008;19: 1625-38

28. Chaloupka JC, Vinuela F, Vinters HV, et al. Technical feasibility and histopathologic studies of ethylene vinyl copolymer (EVAL) using a swine endovascular embolization model. AJNR Am J Neuroradiol 1994;15:1107-15

29. Murayama $Y$, Vinuela F, Ulhoa A, et al. Nonadhesive liquid embolic agent for cerebral arteriovenous malformations: preliminary histopathological studies in swine rete mirabile. Neurosurgery 1998;43: 1164-75

30. Takao H, Murayama Y, Yuki I, et al. Endovascular treatment of experimental aneurysms using a combination of thermoreversible gelation polymer and protection devices: feasibility study. Neurosurgery 2009;65:601-09; discussion 609 